\title{
Possible evidence of gravity wave coupling into the mid- latitude $F$ region ionosphere during the SEEK campaign
}

\author{
M. J. Taylor \\ Space Dynamics Laboratory and Physics Department, Utah State University, Logan
}

J.-M. Jahn

Los Alamos National Laboratory, Los Alamos, NM

\section{S. Fukao, and A. Saito}

Radio Atmospheric Science Center, Kyoto University, Kyoto, Japan

\begin{abstract}
On five of eight observation nights during the 1996 SEEK (Sporadic E Experiment over Kyushu) campaign, Japan, unusual "wave-like" structures were imaged in the $630 \mathrm{~nm}$ thermospheric nightglow emission. Four of these events were observed to travel towards the southwest, providing new evidence in support of recent theories describing the coupling of mediumscale gravity waves into the mid-latitude $F$ region ionosphere. Available ionosonde data and the visual characteristics of the wave structures indicate no association with the occurrence of mid-latitude spread F or F region upwellings. Instead, the data support the novel concept of feedback from the ionosphere into the gravity wave, via the Perkins instability, to enhance its visibility.
\end{abstract}

\section{Introduction}

The $630 \mathrm{~nm}$ thermospheric nightglow emission is host to a variety of $F$ region phenomena and has been the subject of numerous investigations by radio and optical techniques. At high latitudes the emission is dominated by soft auroral particle precipitation while at low latitudes the occurrence of equatorial spread F (ESF) can give rise to extensive field aligned structuring in the form of equatorial depletions [e.g. Weber et al., 1978]. In contrast, observations of the mid-latitude $630 \mathrm{~nm}$ emission have revealed a relative paucity of "structure", most of which is due to the penetration of high and low latitude phenomena into the mid-latitude $\mathrm{F}$ region during disturbed periods [e.g. Mendillo et al., 1987].

The phenomenon of mid-latitude spread $F$ is well documented. It exhibits a peak occurrence in the summer months and medium scale gravity waves are thought to play a major role in its development [e.g. Kelley and Fukao, 1991]. Such waves in the form of traveling ionospheric disturbances (TIDs) are prevalent at midlatitudes, exhibiting periods of several tens of minutes. However, optical measurements of TIDs in the neutral atmosphere are comparatively rare and only very recently have their spatial signatures in the mid-latitude

Copyright 1998 by the American Geophysical Union.

Paper number 97GL03448.

0094-8534/98/97GL-03448\$05.00
$630 \mathrm{~nm}$ airglow been reported [Mendillo et al., 1997; Miller et al., 1997]

In 1991 Fukao et al. described the results of a novel series of measurements using the MU VHF radar at Shigaraki, Japan $\left(34.9^{\circ} \mathrm{N}, 136.1^{\circ} \mathrm{E}\right)$ to investigate midlatitude spread $\mathbf{F}$. The observations were made during the summer months near solar minimum and on several occasions strong turbulent upwellings caused by intense 3 -m field aligned irregularities were observed in association with spread F. The irregularities achieved heights of several hundred kilometers and exhibited clear similarities in form to the radar echo patterns detected at equatorial latitudes in association with ESF. Under exceptional circumstances ESF itself can be detected at mid-latitude sites. If upwellings in the form of "plumes" or "bubbles" rise to extreme heights over the equator then associated depletions in the $630 \mathrm{~nm}$ airglow can extend to locations far away from the magnetic equator. At mid-latitudes the possibility therefore exists of detecting either gravity wave seeding during episodes of mid-latitude spread $\mathbf{F}$ or, under exceptional circumstances, of measuring depletion-like structures in association with strong turbulent equatorial upwelling [Fukao et al., 1991; Mendillo et al., 1997]. One clear distinction between depletions observed at the equator and those expected at mid-latitudes is their direction of motion. Equatorial airglow depletions drift with the ambient plasma towards the $\mathrm{E}$ during the evening when they are most conspicuous, whereas the MU radar measurements indicate a westward drift motion at speeds of typically 125 and $185 \mathrm{~ms}^{-1}$ (measured on two occasions).

The SEEK campaign was conducted from southern Japan during August 1996 under near solar minimum conditions and offered an almost ideal opportunity to search for mid-latitude $F$ region phenomena in association with spread F. As part of this campaign all-sky image measurements of the F region $\mathrm{OI}(630 \mathrm{~nm})$ nightglow emission were made together with observations of the near infrared $\mathrm{OH}, \mathrm{OI}(557.7 \mathrm{~nm})$ and $\mathrm{Na}(589.2 \mathrm{~nm})$ nightglow emissions used to study mesospheric gravity waves and their relationship with sporadic-E [e.g. Fukao et al., this issue]. In this paper we present measurements of unusual "wave-like" structures imaged in the $630 \mathrm{~nm}$ emission. The properties of these waves and their possible role in coupling into the ionosphere are discussed. 


\section{Observations}

Monochromatic image measurements of the nightglow emissions were made from Yamagawa Observatory $\left(31.2^{\circ} \mathrm{N}, 130.6^{\circ} \mathrm{E}\right)$, Kyushu, Japan during the period 9 23 August. The imager utilized a high quantum efficiency bare CCD array binned to $512 \times 512$ pixels and a wide angle $\left(180^{\circ}\right)$ telecentric lens arrangement. Images of the $630 \mathrm{~nm}$ emission (filter bandwidth $\sim 2.4 \mathrm{~nm}$ ) were obtained every 5-7 min using an exposure time of $180 \mathrm{~s}$. A background measurement was also recorded at $572.5 \mathrm{~nm}$ to determine the presence of clouds. Details of this instrument are given in Taylor et al. [1997b].

Due to the limited observing conditions during August (associated with frequent clouds and the passage of a typhoon) our measurements were restricted to eight nights of typically 0.5-2 hours in duration. On five of these nights $(9,16,17,18$, and 21 August UT) "wavelike" structures in the $630 \mathrm{~nm}$ emission were detected. Figure 1 shows a sequence of images illustrating the morphology and dynamics of the structures. (Note, for presentation purposes the field of view of these images has been reduced to $165^{\circ}$ to eliminate foreground obstacles.) The data were recorded on 17 August during a brief $\sim 30 \mathrm{~min}$ period (2230 LT/1330 UT). Five elongated bright crests were observed, of which three are evident in each image, progressing uniformly towards the $\sim \mathrm{SW}$ on a heading of $245^{\circ} \pm 5^{\circ}$. The horizontal separation of the crests was $303 \pm 8 \mathrm{~km}$ and their average speed $159 \pm 3 \mathrm{~ms}^{-1}$ indicating an observed period of $31 \mathrm{~min}$ (assuming they are wave motions). The two leading bright crests extended right across the image field indicating a lateral extent of $>2000 \mathrm{~km}$ (assuming an emission altitude of $280 \mathrm{~km}$ appropriate for solar minimum conditions) while the third crest was less extensive. Unfortunately, no further data were obtained after 1400 UT due to clouds.

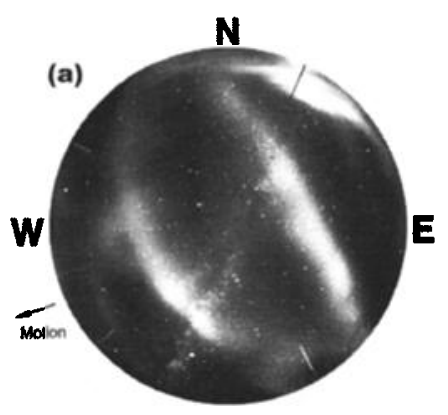

13:28:18 UT

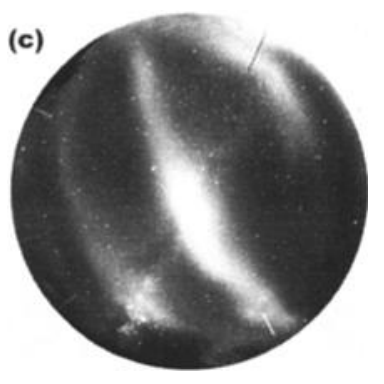

13:44:09 UT

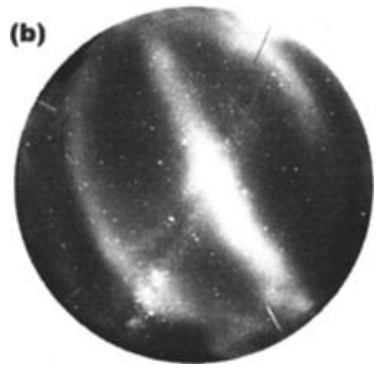

13:39:07 UT

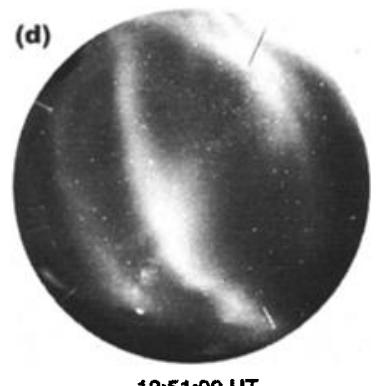

13:51:00 UT
Figure 1. CCD images showing extensive wave-like structures in the $630 \mathrm{~nm}$ nightglow emission recorded on 17 August. Note the progression of three bright crests.

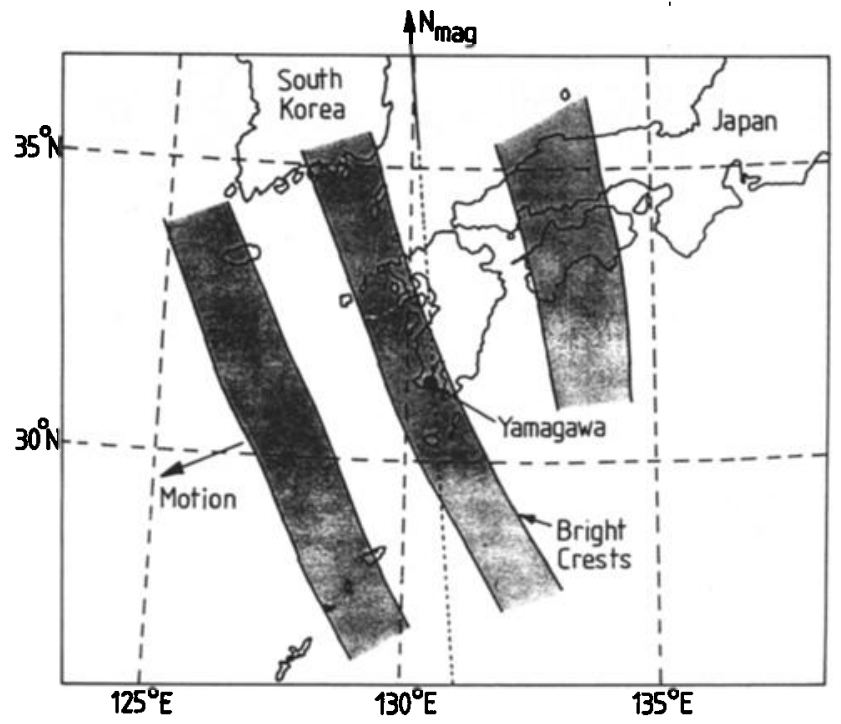

Figure 2. Geographic projection of the three prominent wave forms in Figure 1b for an assumed altitude of $280 \mathrm{~km}$. The direction of wave motion $\left(245^{\circ} \mathrm{N}\right)$ and the orientation of the magnetic field $\left(-5^{\circ}\right)$ are marked.

This event was the most conspicuous of the five $630 \mathrm{~nm}$ displays observed, all but one of which exhibited similar directions of motion towards the $\sim \mathrm{SW}$. On two further nights (19 and 22 August) the sky was clear for a significant period of time and no evidence of wave-like (or other) structures in the $630 \mathrm{~nm}$ emission was found. This indicates a relatively high occurrence frequency of $\sim 60 \%$. Comparison of these data with the gravity wave patterns imaged in the $\mathrm{OH}$ and $\mathrm{OI}(557.7 \mathrm{~nm})$ emissions indicates quite dissimilar horizontal scale sizes and velocities. In particular, most of the mesospheric gravity waves were found to progress towards the $\mathrm{N}-\mathrm{NE}$.

\section{Results}

Figure 2 shows a map of the three bright crests in Figure $1 \mathrm{~b}$ for an assumed altitude of $280 \mathrm{~km}$. The two leading crests clearly exhibit a similar orientation and a direction of motion towards the $\sim \mathrm{SW}$. The third structure appears to be slightly rotated with respect to the other two forms but otherwise it progressed with the same speed. Comparison of the alignment of all three structures with the magnetic meridian at Yamagawa $\left(-5.02^{\circ}\right.$ declination at $280 \mathrm{~km}$ altitude by IGRF 95) suggest a significant difference of $\sim 10^{\circ}-20^{\circ}$. Table 1 summarizes the measured parameters for the five

Table 1. Summary of the $630 \mathrm{~nm}$ wave data, Yamagawa, Japan during the SEEK campaign. (Note, the 17 Aug UT corresponds to a local date of 17/18 Aug etc.)

\begin{tabular}{cccccc}
\hline $\begin{array}{c}\text { UT } \\
\text { Date }\end{array}$ & $\begin{array}{c}\text { UT } \\
\text { Time/Interval }\end{array}$ & $\begin{array}{c}\text { Horizontal } \\
\text { Separation } \\
(\mathrm{km})\end{array}$ & $\begin{array}{c}\text { Horizontal } \\
\text { Speed } \\
(\mathrm{m} / \mathrm{s})\end{array}$ & $\begin{array}{c}\text { Apparent } \\
\text { Periodicity } \\
(\mathrm{min})\end{array}$ & $\begin{array}{c}\text { Direction } \\
\text { from } \mathbf{N}^{\circ}\end{array}$ \\
\hline 9 Aug. & $16: 03-16: 20$ & $292 \pm 15$ & $79 \pm 5$ & 62 & $250^{\circ} \pm 8^{\circ}$ \\
16 Aug. & $12: 35-12: 44$ & $213 \pm 3$ & $93 \pm 10$ & - & $245^{\circ} \pm 7^{\circ}$ \\
17 Aug & $13: 28-14: 00$ & $303 \pm 8$ & $159 \pm 3$ & 31 & $245^{\circ} \pm 5^{\circ}$ \\
18 Aug. & $12: 44-12: 52$ & $187 \pm 20$ & $250 \pm 20$ & 13 & $300^{\circ} \pm 15^{\circ}$ \\
21 Aug. & $13: 26-13: 38$ & $182 \pm 4$ & $47 \pm 3$ & 65 & $255^{\circ} \pm 5^{\circ}$ \\
Average* & & 248 & 95 & & $249^{\circ}$ \\
\hline
\end{tabular}

Note* Averages excluding data from 18 Aug. 
events imaged during the campaign. Four events exhibited directions of motion essentially identical within the measurement error with an average heading of $249^{\circ} \mathrm{N}$. The horizontal scales for all five disturbances ranged from $\sim 180-300 \mathrm{~km}$ and, with the exception of the 18 August event, they all exhibited speeds of typically 50 $160 \mathrm{~ms}^{-1}$, suggesting observed periods $\sim 30-65 \mathrm{~min}$. On 18 August the structures appeared similar in morphology but progressed on a significantly different heading towards the NW and at a substantially higher speed of $250 \mathrm{~ms}^{-1}$. This implies a much shorter period of 13 min, close to the Brunt-Väisälä period at $\mathrm{F}$ region altitudes.

The apparent lack of alignment of these structures with the magnetic meridian; together with the fact that all of the disturbances exhibited a westward component of motion, provides strong evidence that they were not the signatures of extremely high altitude ESF events [Mendillo et al., 1997]. Indeed, as the field lines at Yamagawa map to an apex height of $\sim 1600 \mathrm{~km}$ at the equator (M. Colerico, private communication) such events would be expected to be scarce. In comparison, the properties of the $630 \mathrm{~nm}$ events reported here exhibit several similarities to the mid-latitude $F$ region disturbances reported by Fukao et al. [1991]. The horizontal scale sizes (few hundred $\mathrm{km}$ ), speeds $\left(<200 \mathrm{~ms}^{-1}\right)$ and the westward component of motion are all consistent with mid-latitude upwelling characteristics. Moreover, their detection during the summer months close to solar minimum conditions suggests they may rather be the signature of mid-latitude "depletions".

However, the visual characteristics of the waves in Figure 1 indicate otherwise. A detailed comparison of these data with images of equatorial depletions (such as those reported in Taylor et al. [1997a] which were recorded on the magnetic equator during the Guará campaign using the same imager) indicates a quite dissimilar morphology. Figure 3 shows an intensity scan (plot a) across the three bright crests evident in Figure $1 b$ and a similar length trace (plot b) across several field-aligned ESF depletions evident in Figure 1d of Taylor et al. [1997a]. In the SEEK data the crests appear as brightenings on a dark sky background whereas the Guará data show that the depletions are due to reductions in intensity on an otherwise bright airglow background. This argument is identical to that developed by Mendillo et al. [1997], who associated the unusual observation of wave-like enhancements in $630 \mathrm{~nm}$ image data at Arecibo Observatory, PR $\left(\sim 18^{\circ} \mathrm{N}, \sim 30^{\circ} \mathrm{Nmag}\right)$ with medium scale TIDs. In the Arecibo case the "waves" appear to exhibit similar horizontal scale sizes and phase velocities to those reported here and they were observed in the absence of spread F. Unfortunately, $F$ region data from the MU radar were not available during the SEEK campaign. However, the ionosonde at Yamagawa was operated throughout this period and, despite the presence of strong interference, no sign of range or frequency spreading was evident indicating little of no spread $F$. Thus, the available evidence indicates that the wave patterns imaged during the SEEK campaign were almost certainly not manifestations of mid-latitude spread $F$ but rather were the signatures of thermospheric gravity waves. Ionograms for August 18 and 19 were clear enough to derive density profiles (but not h'F) and on both occasions quasiperiodic variations in the isodensity lines were evident,
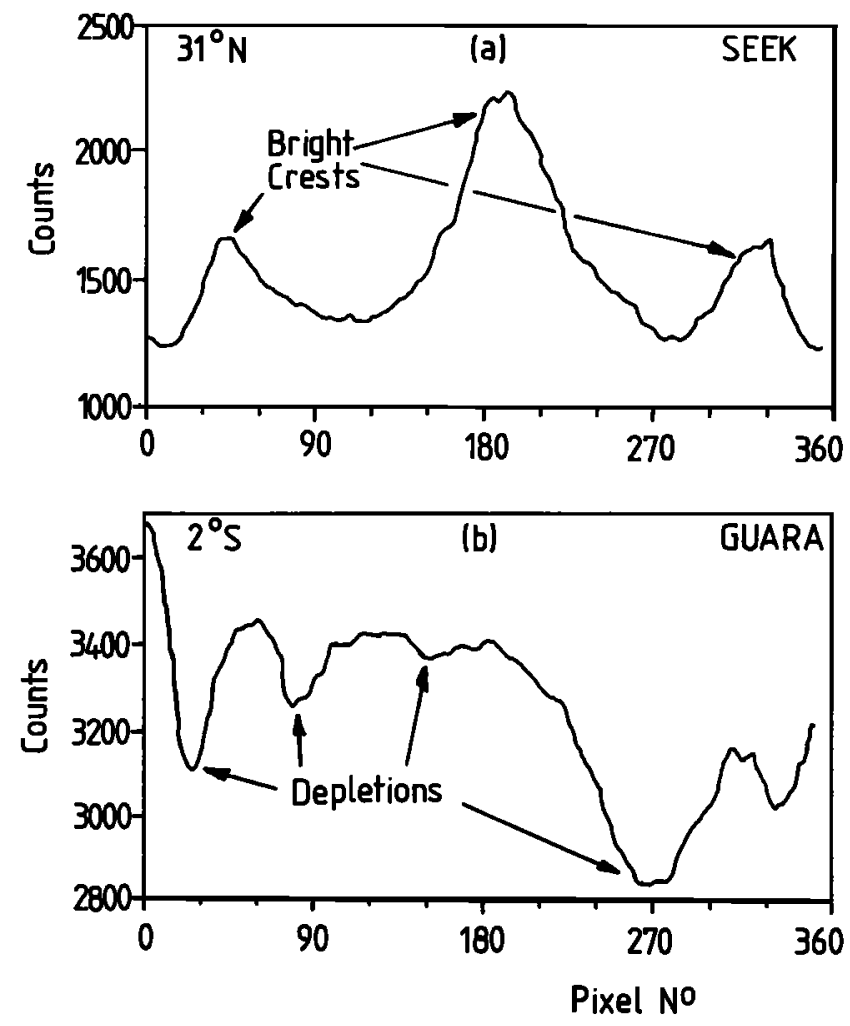

Figure 3. $630 \mathrm{~nm}$ intensity profiles across (a) the three prominent crests of Figure 1b, and (b) several equatorial depletions recorded using the same camera at Alcântara, Brazil $\left(2.2^{\circ} \mathrm{S}\right)$ during the Guará campaign (from Figure 1d of Taylor et al. [1997a]).

suggesting the presence of TIDs. Unfortunately, the data are too sparse to draw further conclusions.

\section{Discussion}

Traditionally, TIDs have been detected in the F region ionosphere using radio techniques whereas optical observations of TIDs are particularly rare (at any latitude). Coordinated optical and radio measurements from Arecibo, discussed in a recent series of articles by Mendillo et al. [1997], Miller et al. [1997] and Kelley and Miller [1997], contain the first such observations of their two-dimensional characteristics. The remarkable similarity between our SEEK data, recorded at a completely different site $\left(\sim 31^{\circ} \mathrm{N}, \sim 26^{\circ} \mathrm{Nmag}\right)$, and the optical measurements at Arecibo is evident. Of particular interest is the common result that almost all of the waves were observed to progress towards the $\sim \mathrm{SW}$. One possible explanation for this is azimuthal filtering of the waves by background winds. However, a more plausible explanation for the anisotropy in wave propagation headings lies in the explanation put forward by the authors of the Arecibo study. They proposed that medium-scale gravity waves propagating through the neutral thermosphere excite an ionospheric response (a locally varying electric field) through the Perkins instability [Perkins, 1973; Miller, 1997]. The Perkins instability is most unstable to rapid growth for gravity waves progressing approximately towards the magnetic W-SW (in the northern hemisphere) as observed by a 
variety of instruments from a range of sites (see Kelley and Fukao [1991]). Such waves may also act as a seed for the development of spread $\mathrm{F}$ and under exceptional circumstances may initiate the generation of turbulent upwellings in the mid-latitude $\mathrm{F}$ region ionosphere.

Miller [1997] and Kelley and Miller [1997] have postulated that such electrodynamic coupling by gravity waves may not only excite an ionospheric response, but that under the right conditions ionospheric disturbances can transfer significant amounts of energy back into the gravity wave. This leads to the notion that gravity waves oriented in directions unstable to the Perkins instability may become significantly enhanced over waves propagating at other azimuths and hence should be detectable more readily. This would explain the observed preference for $630 \mathrm{~nm}$ wave progression towards the $\sim \mathrm{SW}$ in both the Arecibo and now the SEEK data. Our data also show that gravity waves are present at other azimuths, at least sometimes (e.g. 18 Aug event), but that they appear to be significantly fainter and are therefore more difficult to detect. Only recently, with the development of sensitive CCD imaging systems [e.g. Taylor et al., 1997b] are such waves measurable.

\section{Summary}

New measurements of the two-dimensional properties of wave-like structures in the neutral atmosphere at $\mathrm{F}$ region heights $(\sim 280 \mathrm{~km})$ are presented. A strong tendency for wave progression towards the $\sim \mathrm{SW}$ was found on all but one occasion (total 5 events). The $630 \mathrm{~nm}$ wave forms were dissimilar to spread $F$ related depletions in both their visual characteristics and their expected field-aligned orientation (although equatorial depletions have also been observed tilted slightly to the geomagnetic meridian [Weber et al., 1978]). These results are remarkably consistent with the recent reports by Mendillo et al. [1997] and Miller et al. [1997] suggesting that the gravity waves were well oriented to couple efficiently into the ionosphere via the Perkins instability. The observations are also consistent with the concept of feedback from the ionosphere into the gravity wave to enhance its visibility. The apparent lack of strong spread $\mathrm{F}$ during this period suggests that none of these events were successful in exciting a major ionospheric response. Further coincident optical and radar data are critical to determine the abundance and nature of $F$ region gravity waves and their efficiency at coupling into the ionosphere to produce mid-latitude upwellings, as postulated by Kelley and Fukao [1991].

Acknowledgments. We are most grateful to the director and staff at Yamagawa Radio Wave Observatory for the use of their facilities during the campaign and for providing us with the ionosonde data. We thank M. Ishi (Communications Research
Laboratory), S.H. Seo (Utah State University), and M. Colerico (Boston University) for their valuable help. The image measurements were supported by a grant from the Utah Asian Studies Consortium (UCON) and by Kyoto University. The analysis of these data was performed under a National Science Foundation grant No ATM-9525815.

\section{References}

Fukao, S., M.C. Kelley, T. Shirakawa, T. Takami, M. Yamamoto, T. Tsuda, and S. Kato, Turbulent upwelling of the mid-latitude ionosphere 1 . observational results by the MU radar, J. Geophys. Res, 96, 3725-3746, 1991.

Fukao, S., M. Yamamoto, R. Tsunoda, H. Hayakawa, The SEEK (Sporadic-E Experiment over Kyushu) campaign, Geophys. Res. Lett., this issue, this issue.

Kelley, M.C., and S. Fukao, Turbulent upwelling of the midlatitude ionosphere 2. theoretical framework, J. Geophys. Res, 96, 3747-3753, 1991.

Kelley, M.C., and C.A. Miller, Electrodynamics of midlatitude spread F, 3, Electrohydrodynamic waves? A new look at the role of electric fields in thermospheric wave dynamics, J. Geophys. Res., 102, 11539-11548, 1997.

Mendillo, M., J. Baumgardner, J. Aarons, J. Foster, and J. Klobuchar, Coordinated optical and radio studies of ionospheric disturbances: Initial results from Millstone Hill, Ann. Geophys, 5, 543-550, 1987.

Mendillo, M., J. Baumgardner, D. Nottingham, J. Aarons, B. Reinisch, J. Scali, and M. Kelley, Investigations of thermospheric-ionospheric dynamics with 6300-A images from Arecibo observatory, J. Geophys. Res., 102, 7331-7344, 1997.

Miller, C.A., W.E. Swartz, M.C. Kelley, M. Mendillo, D. Nottingham, J. Scali, and B. Reinisch, Electrodynamics of midlatitude spread F, 1, Observations of unstable, gravity waveinduced ionospheric electric fields at tropical latitudes, J. Geophys. Res., 102, 11521-11532, 1997.

Miller, C.A., Electrodynamics of midlatitude spread F,2, A new theory of gravity wave electric fields, J. Geophys. Res., 102, 11533-11538, 1997.

Perkins, F.W., Spread F and ionospheric currents, J. Geophys. Res., 78, 218-226, 1973.

Taylor, M.J., J.V. Eccles, J. LaBelle, and J.H.A. Sobral, High resolution OI(630 nm) image measurements of F-region depletion drifts during the Guará campaign, Geophys. Res. Lett., 24, 1699-1702, 1997a.

Taylor, M.J., W.R. Pendleton Jr., S. Clark, H. Takahashi, D. Gobi, and R.A. Goldberg, Image measurements of short-period gravity waves at equatorial latitudes, J. Geophys. Res., in press, 1997b.

Weber, E.J., J. Buchau, R. Eather, and S.B. Mende, North-south aligned equatorial airglow depletions, J. Geophys. Res., 83, 712-716, 1978.

M. J. Taylor, Space Dynamics Laboratory and Physics Department, Utah State University, Logan, UT 84322-4145, USA; e-mail: mtaylor@cc.usu.edu; Tel: 435-797-3919/3519

J.-M. Jahn, MS D-436 Los Alamos National Laboratory, Los Alamos, NM 87545, USA

S. Fukao, and A. Saito, Radio Atmospheric Science Center, Kyoto University, Kyoto, Japan

(Received July 24, 1997; revised October 1, 1997; accepted November $6,1997$. 